\title{
Novel techniques for image process in electron probe microanalysis
}

\author{
N. Mori, ${ }^{*}$ H. Takahashi, ${ }^{*}$ M. Takakura* and C.Nielsen**
}

*JEOL Ltd., 1-2 Musashino 3-chome, Akishima, Tokyo 196,Japn

**JEOL USA Inc., 11 Dearborn Road Peabody, MA 01960, USA

\section{Introduction}

For observing the element distribution in electron probe microanalysis, X-ray map of dots recorded on photo film has been used for a long time. Recently, with the development of computer technology, color map made by applying various image processing to digital data has appeared in order to emphasize important information, though raw data constituting a lot of information are important for observers.

In this report, we will introduce an image processing to smooth the rough outline by increasing pixel numbers without changing original number of pixels. Further, we will introduce a method of correcting the X-ray spread due to electron scattering in the sample by means of sharpness processing.

\section{Principle and results}

Sometimes, as a result of measurement time, saving, the data points become insufficient for map analysis. This leads to a low resolution map. In such case, the image quality can occasionally be improved by "bicubic method", which is a method to increase the pixel number artificially.

The bicubic method is illustrated in Fig. 1, where the digital data of neighboring points are connected by a curved line made of cubic polynomial. In the actual image processing, as each data point has four neighboring points, curved surface passing though the data points can be formed. Thus, we can increase the pixel number for image processing by interpolating the observed data. This improves the image quality.

Fig. 2 is an X-ray map of $\mathrm{Fe}$ in a ceramic. A) is the image of $60 \times 60$ pixels. B) is the image of $240 \times 240$ pixels. The data acquisition time is equal. In the image A) dots at the analysis points stand out, and in image B) the $\mathrm{S} / \mathrm{N}$ ratio is bad. $\mathrm{C}$ ) is the image made by applying the bicubic method to the image A). The pixel number is 240x240.The edge image becomes smooth without lengthening the data acquisition time.

The space resolution of X-ray map is almost $1 \mu \mathrm{m}$ as a result of electron scattering in the sample. We will report a newly developed sharpness processing to correct the X-ray spread carried by electron scattering. 


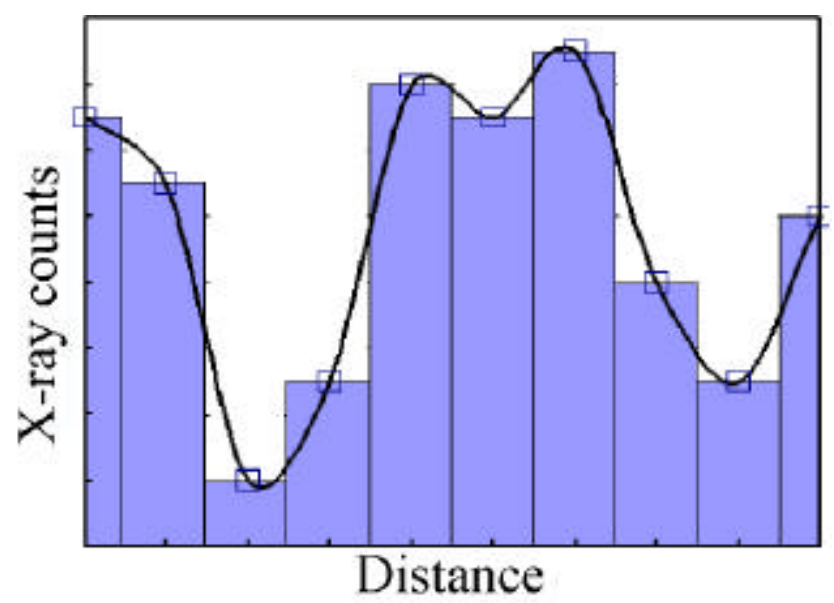

Fig. 1 Conceptual diagram of bicubic method. Digital data at the analysis points are connected by a curved line.

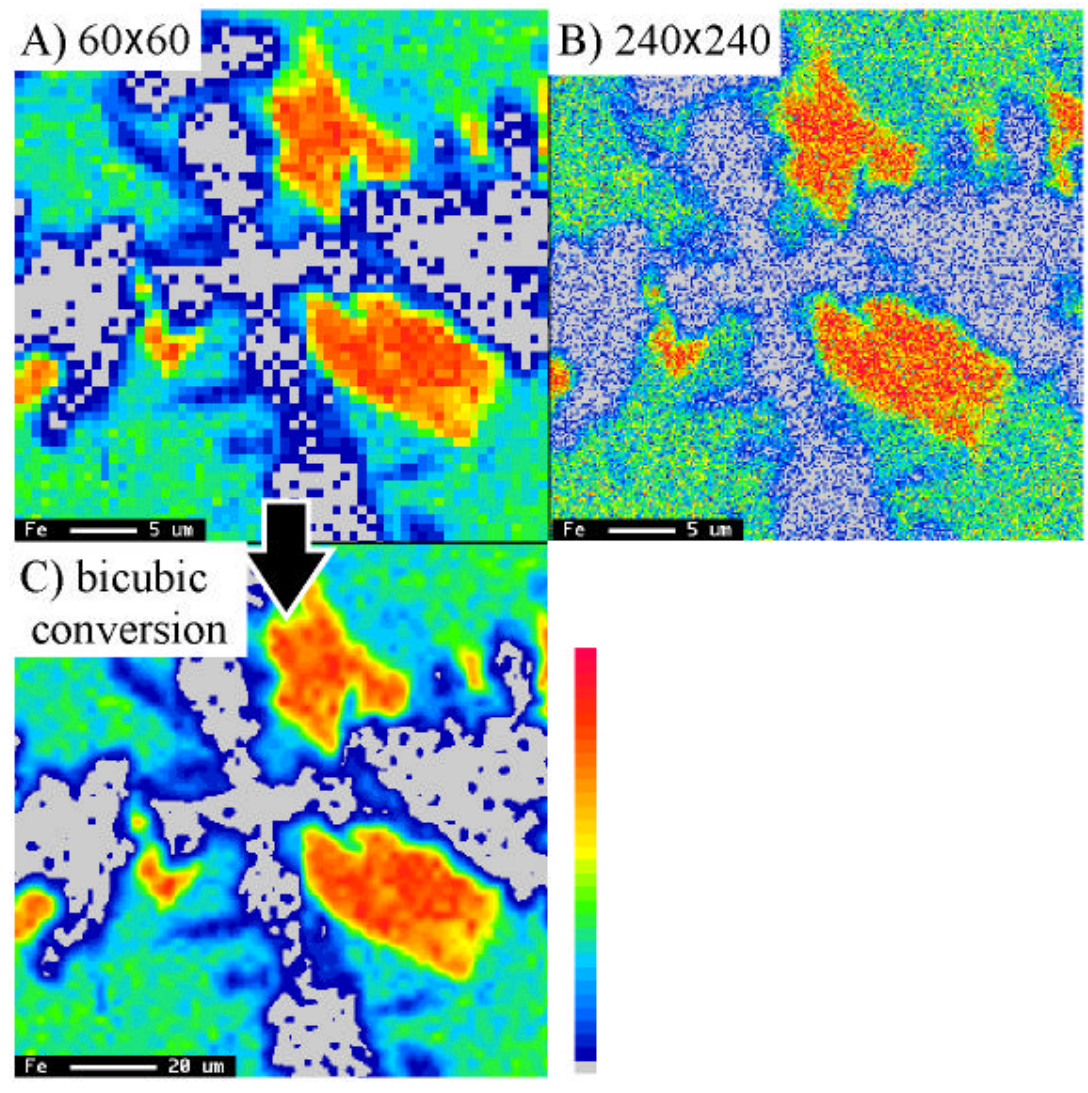

Fig. 2 X-ray map of Fe in a ceramics.
A): $60 \times 60$ pixels.
B): $240 \times 240$ pixels. Analysis times is equal in A).
C): Application of bicubic method. $240 \times 240$ pixels.. 